Journal Of Al-Azhar University Engineering Sector

Vol. 14, No. 51, April, 2019, 543-551

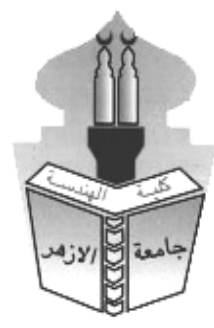

\title{
POWER SYSTEM STABILITY ENHANCEMENT BY PV DISTRIBUTED GENERATION
}

\begin{abstract}
Abdelmoezz Ahmed Eid, M. A. A. Mehanna and Tarek Mahmoud
Dept. of Electrical Engineering, Al-Azhar University, Cairo, Egypt

\section{ABSTRACT}

the present paper investigates the effect of penetration of photovoltaic generation (PVGs) systems on the transient and small signal stability. Recently interest in photovoltaic (PV) power generation systems is increasing rapidly and the installation of large PV systems or large groups of PV systems that are interconnected with the power system grid is accelerating despite their high cost and low efficiency due to environmental issues and depletions of fossil fuels. Photovoltaic generators (PVGs) have significant impacts on the existing power systems, these impacts may be either positively or negatively depending on many factors such as PVGs location, size and inverter characteristics. The eigenvalue analysis and the transient analysis of the system are done without any PVGs penetration and with PVGs penetration and then the results are compared. The investigation is applied on IEEE-39 bus test system. The stability analysis is carried out using simulation program of power system analysis tool box (PSAT).
\end{abstract}

Keywords : Photovoltaic generators (PVGs), Small signal stability, PSAT, Eigen values, Transient stability

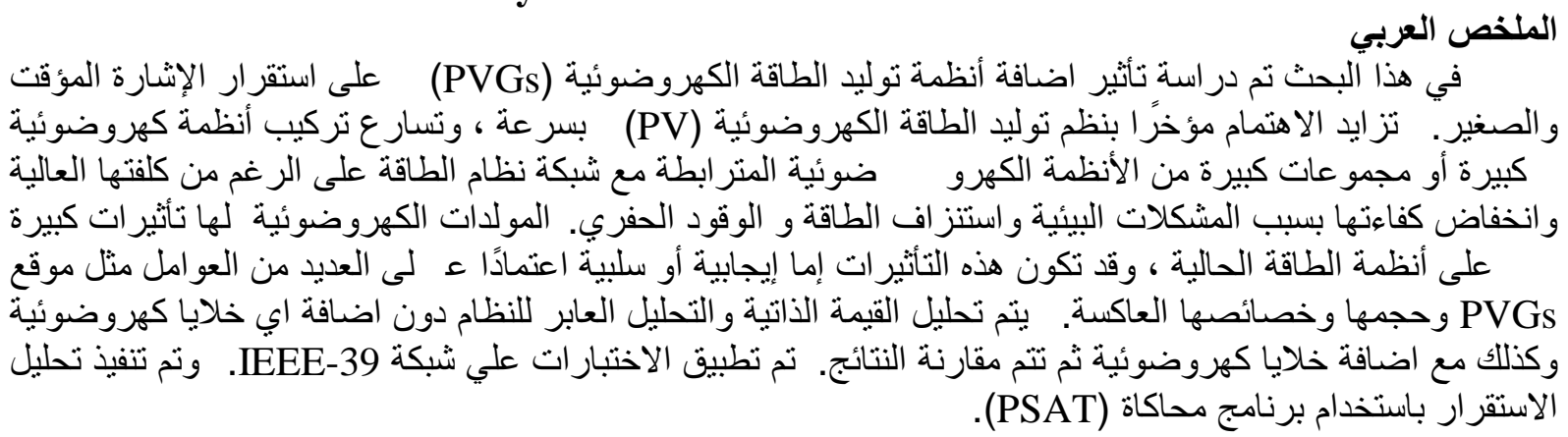

\section{INTRODUCTION}

With increasing the demand of using electricity so the conventional power plant for generating electricity can't be provide this huge amount of demanded electricity specially that depended on the fossil fuel which be a finite reserve [1].

So the world trend to produce the electricity from renewable energy resources such as solar power to overcome the disadvantages of the conventional power plants such as limited reserve and environmental effects as the greenhouse effect caused by the increase of the $\mathrm{CO}_{2}$ concentration in the earth's atmosphere problem [2].

The integration of Photovoltaic Generators (PVGs) in power system networks has a large effect not only on the distribution networks but also on the national transmission and generation system [3].

High PV penetration levels can significantly affect the steady state as well as the transient stability of the systems due to their distinct characteristics that differ from conventional generation resources. With high PV generation, a significant amount of conventional 
generation may be replaced with distributed PV resources. While a portion of this replaced generation is supplied by utility scale PVs, a majority of PV generation addition is expected to be provided by residential rooftop PVs that are located closer to the loads on the distribution system [4].

This paper is organized as follows. Section 2 describes the concept of the small signal stability analysis. Section 3 gives the description of the test system and the modeling of the PVGs system. Section 4 describes analysis of the small signal and transient analysis of the system. And finally conclusions which are drawn from the analysis are given in the section 5.

\section{SMALl SIGNAL STABILITY}

The behavior of power system dynamic can be described by a set of $n$ first order nonlinear ordinary differential equations in vector-matrix notation [5]

$\dot{\mathrm{X}}=\mathrm{f}(\mathrm{x}, \mathrm{u}, \mathrm{t})$

Where $\mathrm{x}=\left(\mathrm{x}_{1}, \mathrm{x}_{2} \ldots . . \mathrm{x}_{\mathrm{n}}\right)^{\mathrm{T}}$ is the vector of state variables, $\mathrm{u}=\left(\mathrm{u}_{1}, \mathrm{u}_{2}, \ldots . \mathrm{u}_{\mathrm{r}}\right)^{\mathrm{T}}$ is the vector of system input variables.

$\mathrm{Y}=\left(\mathrm{y}_{1}, \mathrm{y}_{2}, \ldots . \mathrm{y}_{\mathrm{m}}\right)^{\mathrm{T}}$

Where $Y$ is the vector of system outputs variables, $f=\left(f_{1}, f_{2}, \ldots . f_{n}\right)^{T}$ and $g=\left(g_{1}, g_{2}, \ldots . g_{m}\right)^{T}$ are the vectors of nonlinear functions defining the states and the outputs respectively of the system, time is denoted by $\mathrm{t}$, and the derivative of sate variable $\mathrm{X}$ with respect to time is $\dot{X}$.if the derivative of the state variables are not explicit function of the time, equations (1) and (2) can be simplified as:

$\dot{X}=f(x, u), Y=g(x, u)$

For small signal stability analysis a small perturbation is considered, the non-linear function $\mathrm{f}$ and $g$ can be linearized using Taylor series with the initial points $\quad \mathrm{x}=\mathrm{x}_{0}$ and $\mathrm{u}=\mathrm{u}_{0}$,

The system can express in the following equation:

$\Delta \dot{\mathrm{x}}=\mathrm{A} \Delta \mathrm{x}+\mathrm{B} \Delta \mathrm{u}, \quad \Delta \mathrm{y}=\mathrm{C} \Delta \mathrm{x}+\mathrm{D} \Delta \mathrm{u}$

Where $\Delta \mathrm{x}$ is a small deviation in the state vector, $\Delta \mathrm{y}$ is a small deviation in the output vector, $\mathrm{A}$ is the state matrix, $\mathrm{B}$ is the input matrix, $\mathrm{C}$ is the output coefficient matrix and $\mathrm{D}$ is the feed forward matrix [6].

According to Lyapunov's first method, the eigenvalues of the state matrix A can be illustrate the behavior of the system according to small signal stability, the eigenvalues of the state matrix A may be:

1-A real eigenvalue corresponds to a non- oscillatory mode where a negative real Eigen value represents a decaying mode, a positive real represents aperiodic instability.

2-Complex eigenvalues occur in conjugate pairs, and each pair corresponds to an oscillatory mode.

- When the Complex eigenvalues have negative real parts, the original system is stable.

- When at least one of the Complex eigenvalues has a positive real part, the original system is unstable.

- When at least one of the eigenvalues has zero value, the original system is critical stable [7].

For any eigenvalue $\lambda_{\mathrm{i}}$, the n-column vector $\Phi_{\mathrm{i}}$ is called the right eigenvector which gives the mode shape and the n-row vector $\Psi_{i}$ is called the left eigenvector identifies which combination of the original state variables displays only the $i^{\text {th }}$ mode , are satisfies Equations:

$\mathrm{A} \Phi_{\mathrm{i}}=\lambda \Phi_{\mathrm{i}}$

$\Psi_{\mathrm{i}} \mathrm{A}=\lambda_{\mathrm{i}} \Psi_{\mathrm{i}}$ 
Where $\Psi_{\mathrm{i}} \Phi_{\mathrm{i}}=1$

A measure of the association between the state variables and the modes is the participation factors, $p=\left[p_{1} p_{2} \ldots \ldots p_{n}\right]$, with

$$
\mathrm{Pi}=\left[\begin{array}{c}
\mathrm{P} 1 \mathrm{i} \\
\mathrm{p} 2 \mathrm{i} \\
\vdots \\
\mathrm{pni}
\end{array}\right]=\left[\begin{array}{c}
\Phi 1 \mathrm{i} \Psi \mathrm{i} 1 \\
\Phi 2 \mathrm{i} \Psi \mathrm{i} 2 \\
\vdots \\
\Phi \text { ni } \Psi \text { in }
\end{array}\right]
$$

\section{DESCRIPTION OF THE TESTED SYSTEM}

\section{A. IEEE-39 Bus System.}

IEEE-39 bus system is used for the analysis of the PVGs penetration. The total load of the system is $6586 \mathrm{MW}$. There are ten generators in the system connected at buses from 30 to 39 as shown in the following fig. (1). the ten generators are $6^{\text {th }}$ order and equipped with IEEE type II governors and IEEE type II exciters.

In this paper, the utility scale PV plant will be added into the system to see the impact of the PV system on the small signal and transient stability in addition to conventional synchronous generators.

The PV penetration level in the system is defined as,

PV Penetration $(\%)=($ Total PV generation $(\mathrm{MW}) \div$ Total generation $(\mathrm{MW})$

\section{B. Modeling of PVGs Systems}

The single-line diagrams of the developed PVGs models according to the control modes and their capabilities are depicted in Figs.2 and .3 , for Model 1 (constant PQ) and Model 2 (constant PV), respectively.

There are various possibilities for inverter transfer functions; however, the following two are probably the most appropriate [8]:

(a) First order functions with unity steady state gain.

(b) The closed loop controller transfer functions.

Both yield very similar results and hence the first one is adopted here. Figs. 2 and 3 show the block-diagrams of the two developed models.

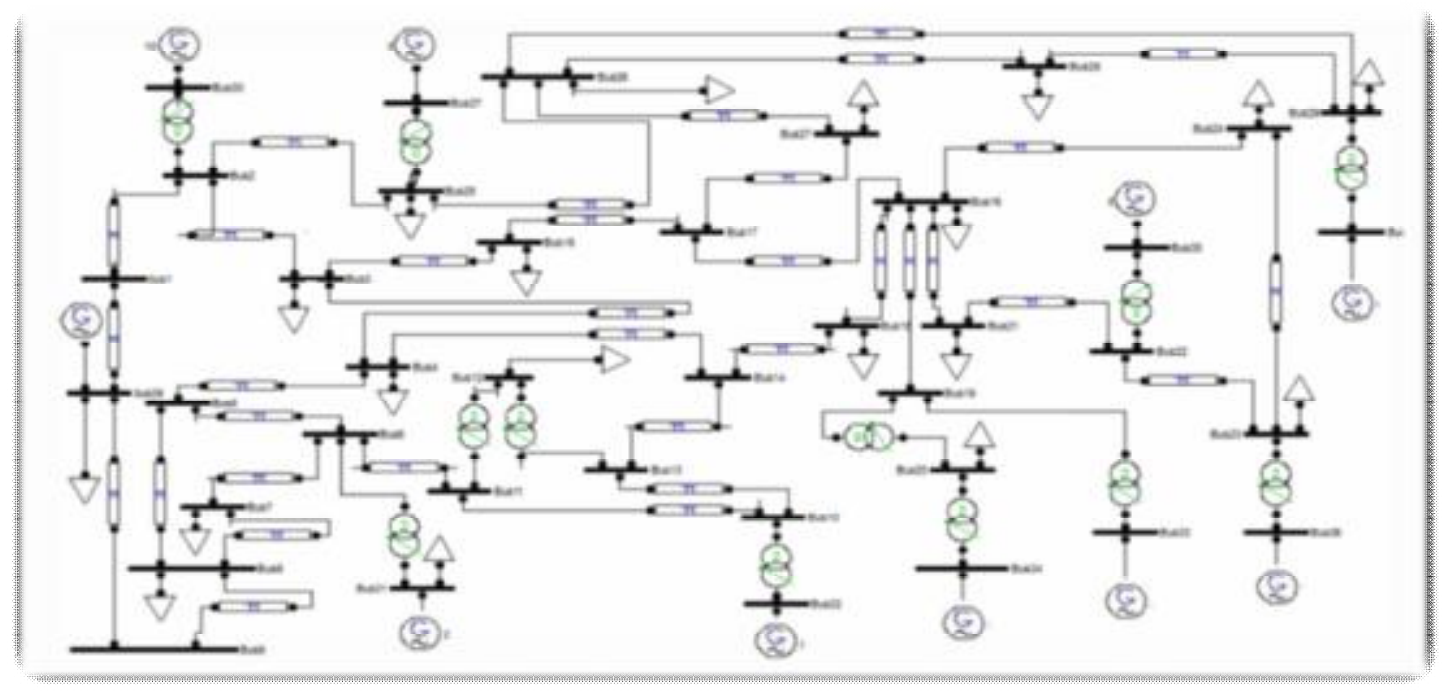

Fig.1. IEEE-39 bus test system 


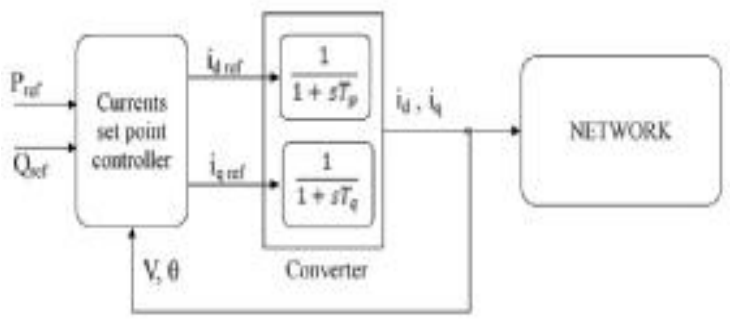

fig.2. PVGs Model 1 block diagram

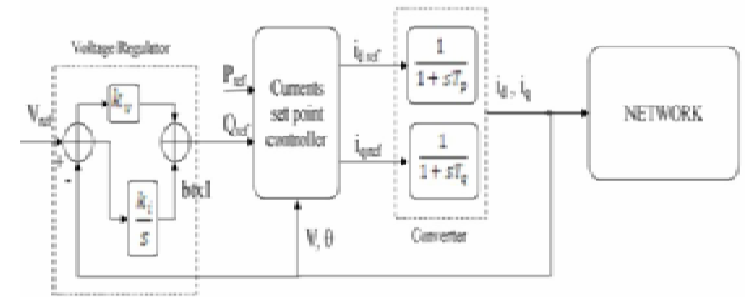

fig. 3. PVGs Model 2 block diagram

In these models, the current set points can be obtained based on the desired active and reactive powers and current measurements of terminal voltage in the dq reference frame.

In Fig. 3, the reference value for reactive power is obtained based on the set-point and actual voltage values through a PI controller [9].

\section{Simulation AND RESUlts}

\section{A. Eigenvalue Analysis}

The objective of the paper is to analyze the small signal stability of the system with and without adding PVGs into the system. For this using PSAT program, depending on the Eigenvalues of the system the following scenario is implemented.

$\underline{C A S E(1)}$ Represents the base case where all generators in the system are synchronous generator and there is no PVGs in the system.

a) Normal case.

In this case, the system operates at its rated load. Small signal stability of the tested system is computed and Eigenvalues of the system with its dominant states as shown in table.1 and Fig.4.

TABLE 1. Computed Eigenvalues of base case

\begin{tabular}{|c|c|c|c|}
\hline \multicolumn{2}{|c|}{ Zero Eigen } & \multicolumn{2}{c|}{ Positive Eigen } \\
\hline number & Dominant states & number & Dominant states \\
\hline 91 & delta_Syn_9 & -- & -- \\
\hline
\end{tabular}

As seen, IEEE-39 bus test system has 110 Eigen number all Eigen numbers are negative except the Eigen number $\lambda 91$ is zero value, so the system is critically stable. The participation factors associate the delta of synchronous generator connected at bus 39 with this critically stable of the system.

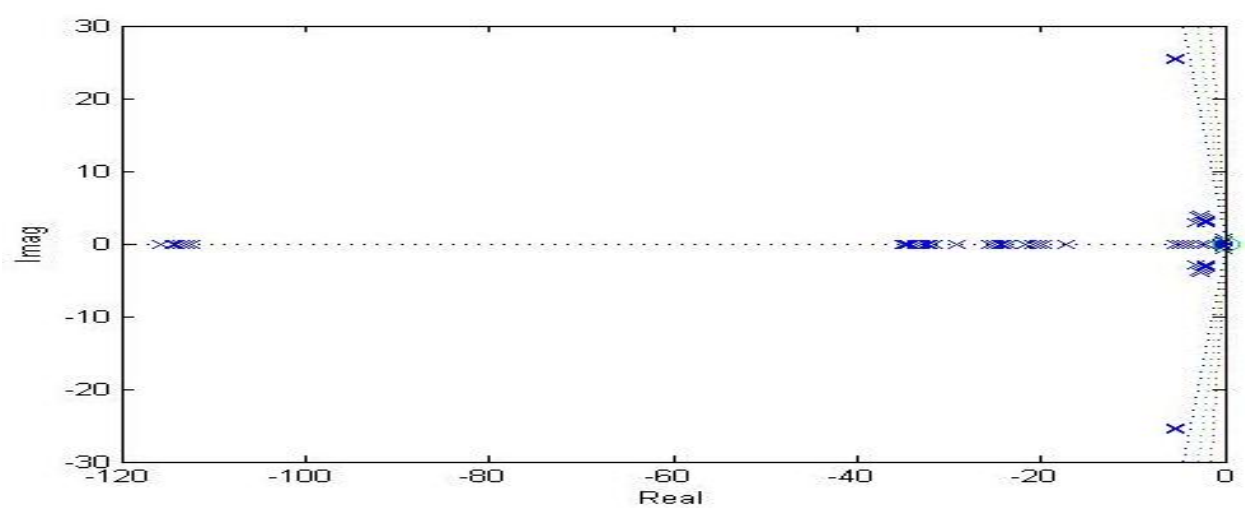

fig. 4. Computed Eigen values of base case

\section{b) Contingency case}

In this case the system loads are increased into $120 \%$ of its rated values and compute the small signal stability of the system; Eigenvalues of the system with its dominant states are shown in table. 2 and Fig.5. 
TABLE 2. Computed Eigenvalues of contingency base case

\begin{tabular}{|c|c|c|c|c|}
\hline \multirow{2}{*}{ system } & \multicolumn{2}{|c|}{ Zero Eigen } & \multicolumn{2}{|c|}{ Positive Eign } \\
\cline { 2 - 4 } & number & Dominant states & number & Dominant states \\
\hline $\begin{array}{c}\text { Increasing system } \\
\text { load into 120\% }\end{array}$ & 94 & delta_Syn_2 & 61 & delta_Syn_1 \\
vr2_Exc_1
\end{tabular}

As shown, all Eigen numbers are negative except the Eigen number $\lambda 94$ is zero value and Eigen numbers $\lambda 61$ and $\lambda 91$ are positive real parts. So the system is unstable.

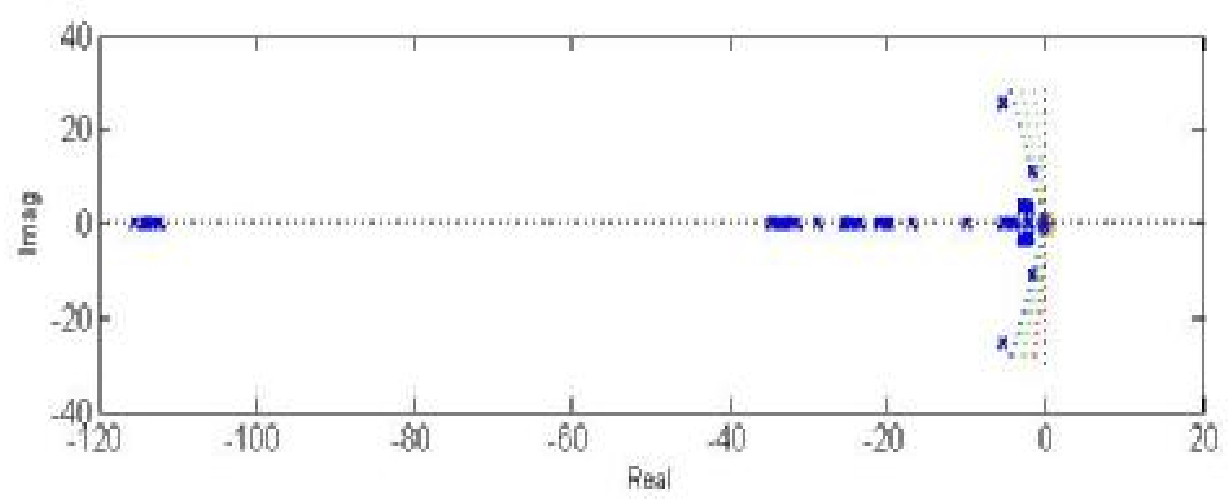

fig. 5. Computed Eigen values of Contingency case

$\underline{C A S E(2)}$ Adding PVGs into the system. According to [10], the optimal location to construct PVGs with ratio $10 \%$ of the total load is at bus 8 .

a) Normal case

Small signal stability test is done; Eigenvalues of the system with its dominant states are shown in table.3.

TABLE 3. Computed Eigenvalues of system with $10 \%$ PVGs.

\begin{tabular}{|c|c|c|c|}
\hline \multicolumn{2}{|c|}{ Zero Eigen } & \multicolumn{2}{|c|}{ Positive Eigen } \\
\hline number & Dominant states & number & Dominant states \\
\hline 95 & delta_Syn_10 & -- & -- \\
\hline
\end{tabular}

The results show that the system has 113 Eigen number all Eigen numbers are negative except the Eigen number $\lambda 95$ is zero value so the system is critically stable. The participation factors associate the delta of synchronous generator connected at bus 30 with this critically stable of the system.

\section{b) Contingency case}

In this case the system loads are increased into $120 \%$ of its rated values and compute the small signal stability of the system; Eigenvalues of the system with its dominant states are shown in table.4.

TABLE 4. Computed Eigenvalues of Contingency system with $10 \%$ PVGs.

\begin{tabular}{|c|c|c|c|c|}
\hline \multirow{2}{*}{ system } & \multicolumn{2}{|c|}{ Zero Eigen } & \multicolumn{2}{c|}{ Positive Eigen } \\
\cline { 2 - 5 } & number & Dominant states & number & Dominant states \\
\hline $\begin{array}{c}\text { Increasing system } \\
\text { load into 120\% }\end{array}$ & 97 & delta_Syn_10 & -- & -- \\
\hline
\end{tabular}

As shown, all Eigen numbers are negative except the Eigen number $\lambda 97$ is zero value. So the system is critically stable. The participation factors associate the delta of synchronous generator connected at bus 30 with this critically stable of the system. 


\section{B. Transient analysis}

For IEEE 39 bus system a fault is making at bus 12 . This fault is three phase fault occurs at $t$ $=1 \mathrm{sec}$ and its duration is $0.25 \mathrm{~s}$ then the fault is clearing. The effect of this fault on voltages of nearing buses, speeds and power angles of generators and active and reactive powers of generation buses when there's PVGs with rating $658.8 \mathrm{MW}$ at bus 8 and there's no PVGs as shown in the following figures.

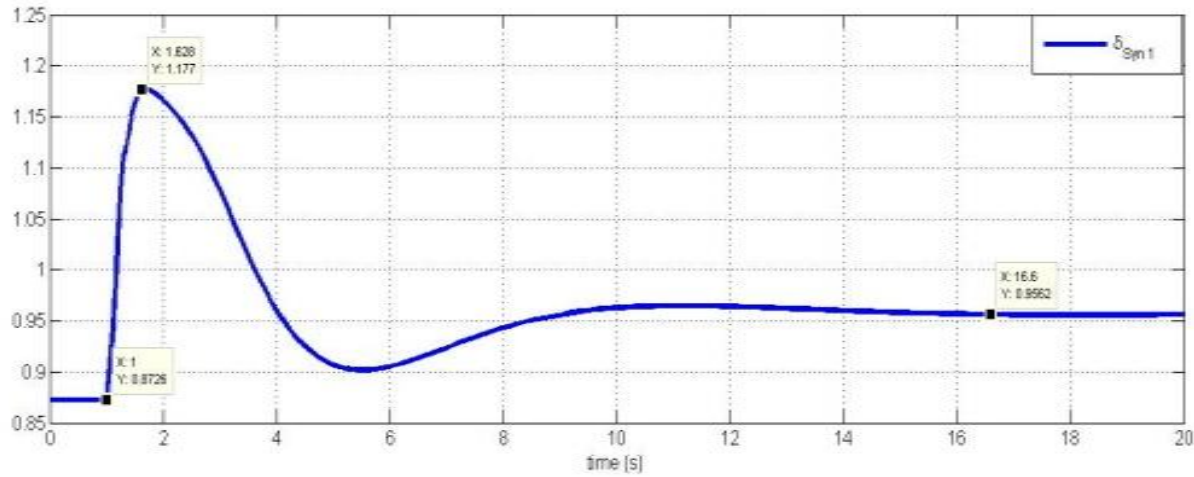

fig. 6. Rotor angles of the slack generator without PV penetration

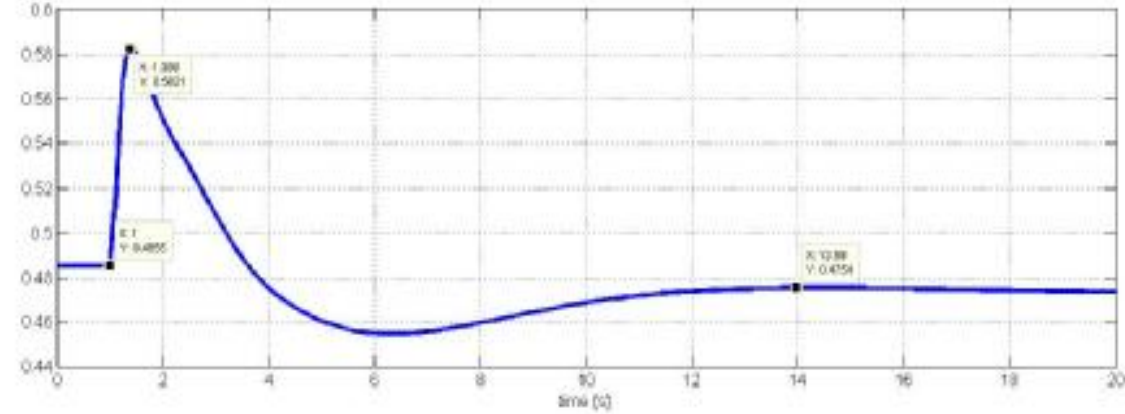

fig. 7. Rotor angles of the slack generator with $10 \% \mathrm{PV}$ penetration

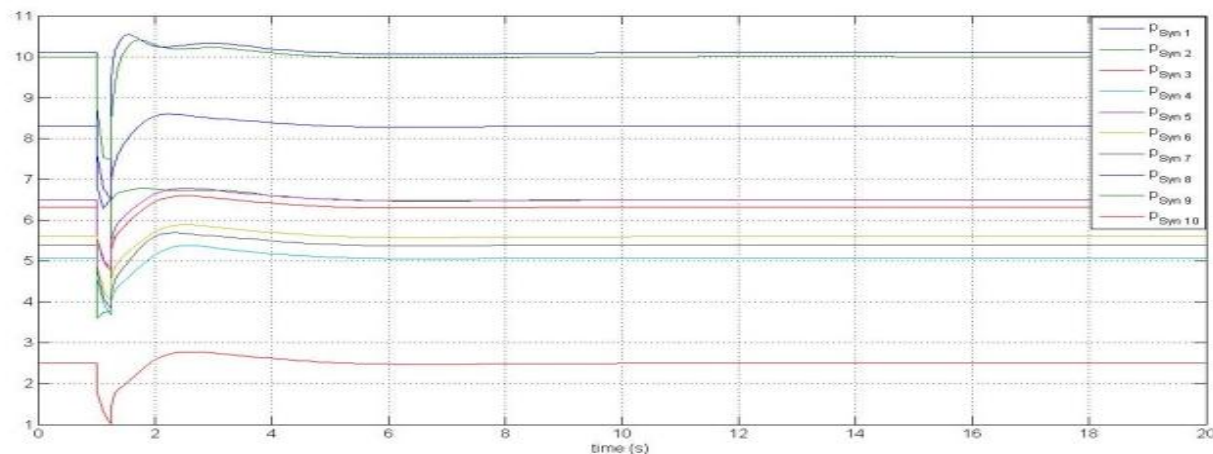

fig.8. Active power of generators without PV penetration

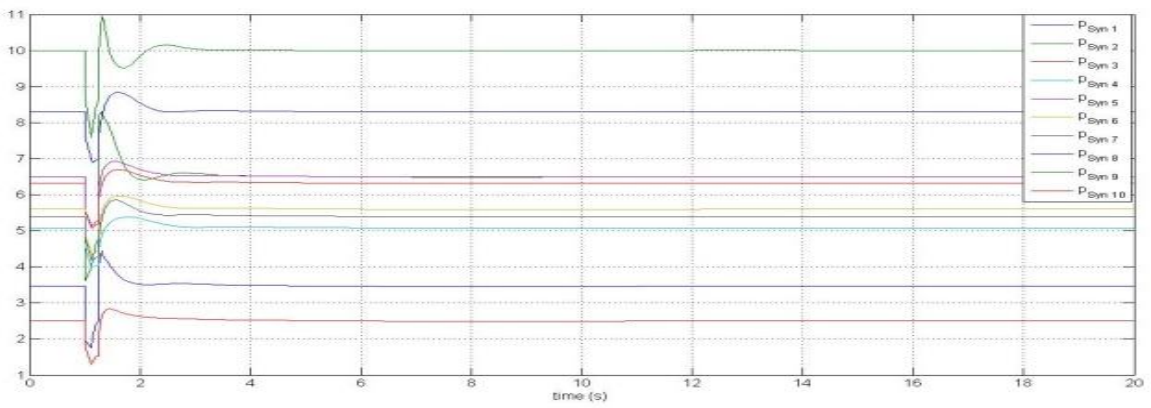

fig. 9. Active power of generators with $10 \% \mathrm{PV}$ penetration 


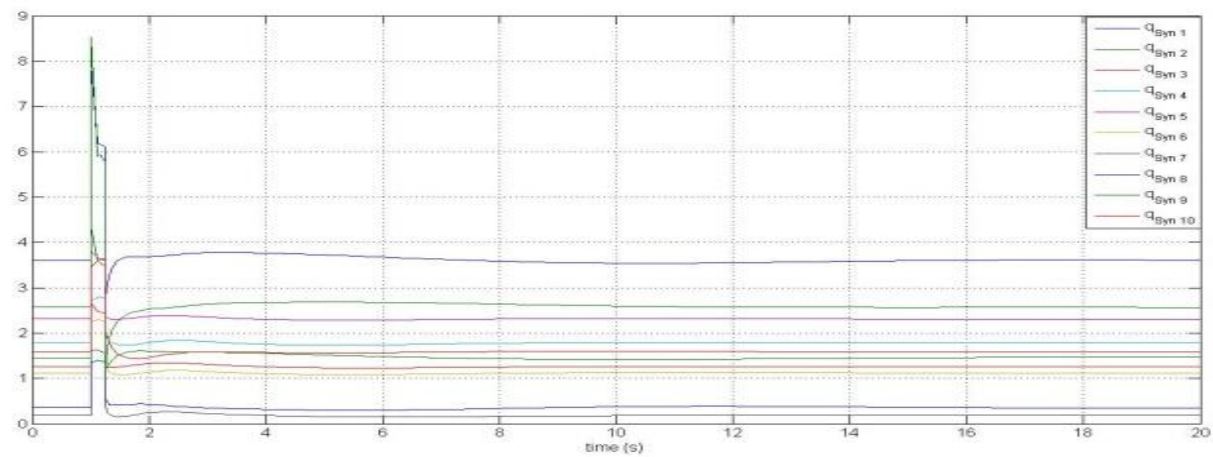

fig.10. Reactive power of generators without PV penetration

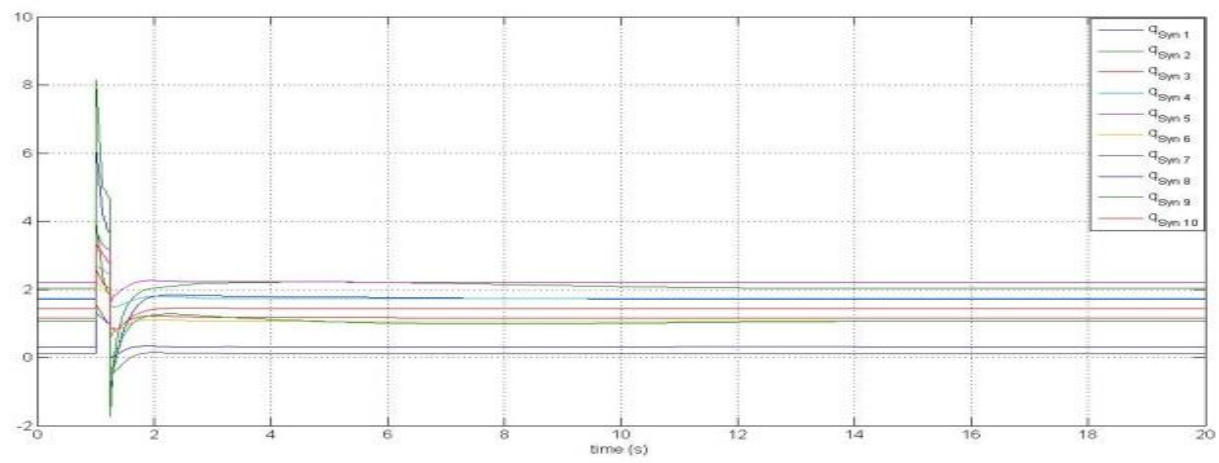

fig. 11. Reactive power of generators with $10 \% \mathrm{PV}$ penetration

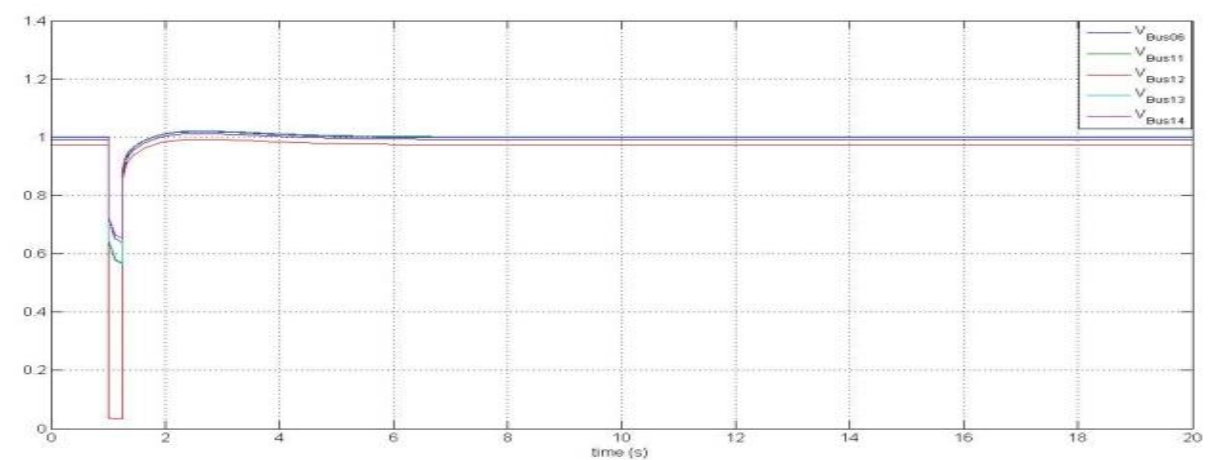

fig. 12. Voltages at nearing buses to bus 12 without PV penetration

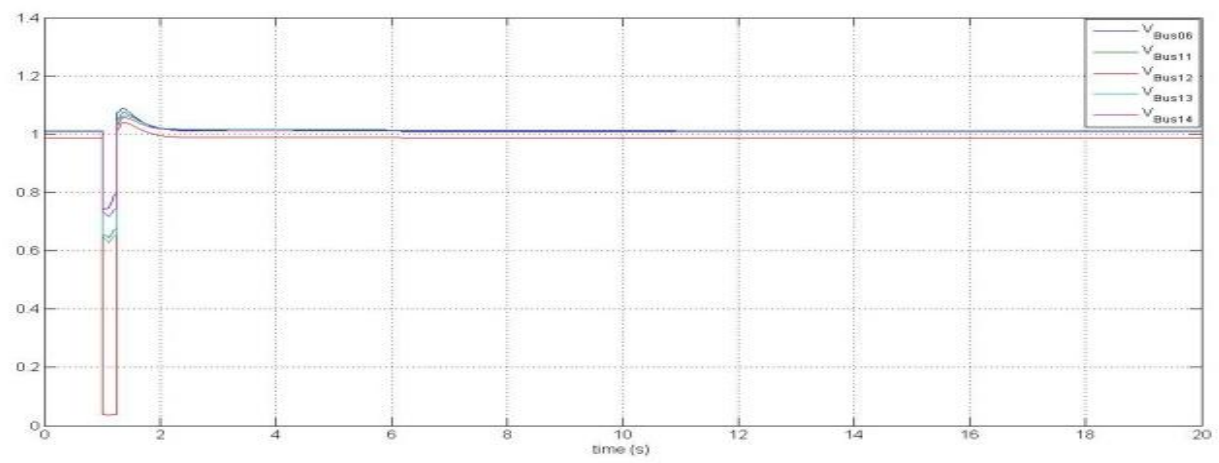

fig. 13. Voltages at nearing buses to bus 7 with $10 \%$ PV penetration 


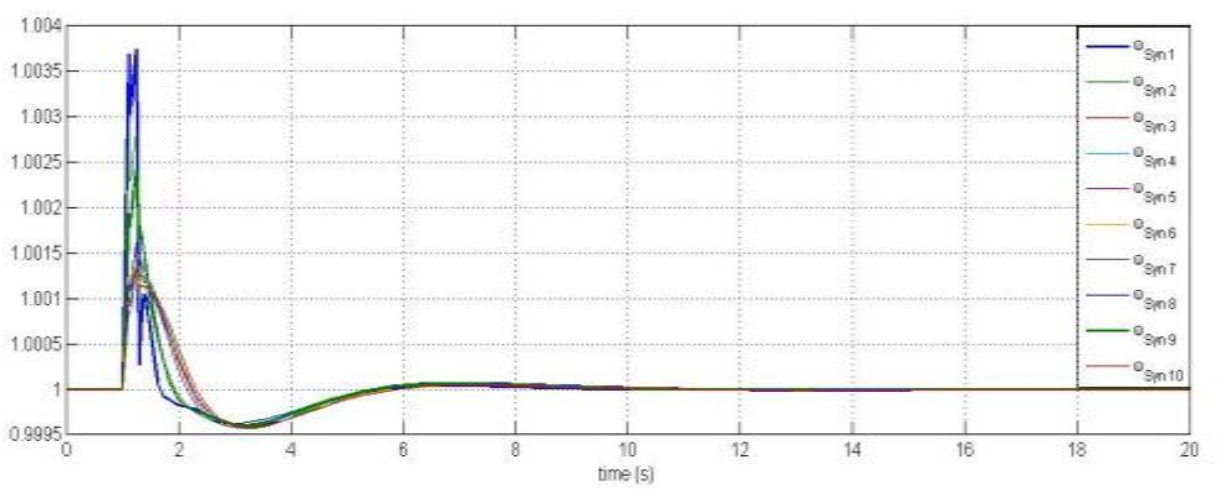

fig.14. Speeds of generators without PV penetration

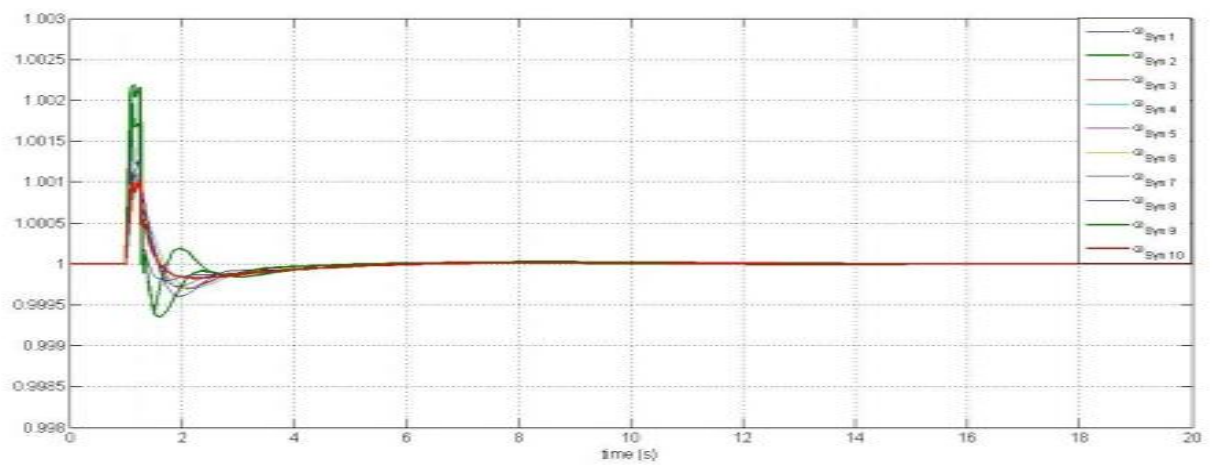

fig. 15. Speeds of generators with $10 \% \mathrm{PV}$ penetration

As seen in figures 6 to 15, power angle of the slack synchronous generator witch connected at bus 31, active and reactive power of generators, voltages at nearing buses to bus 12 and the speeds of generators are plotted during transient disturbance. When PVGs are connected to the system, the curves are stabilized faster than the case which no PVGs are connected to the system.

From all the previous studied cases, adding of the PV generation leads to more stable system in addition to the other advantages of solar energy.

\section{CONCLUSION}

Is this paper, for IEEE 39-bus power system the investigation of the small signal stability analysis and Transient stability analysis has been done with and without injection of Photovoltaic generation. To observe the effect of Photovoltaic Generation on the small signal stability of the system, the eigenvalue sensitivity has been used considering increase of the load of the system. It showed that adding of PVGs into the system leads to improvement in small signal stability of the system. Transient stability analysis performed through time domain simulations, shown that the system contains PVGs are able to restore rotor angles, voltages, speeds and power after a three phase fault better than conventional generators though a quick response in restoration . 


\section{REFERENCES}

1. Yun Tiam Tan, "Impact on the Power System with a Large Penetration of Photovoltaic Generation", Umist, February 2004..

2. Thomas Ackermann," Wind Power in Power Systems", John Wiley \& Sons, Ltd, England, 2005.

3. Sara Eftekharnejad, "The Impact of Increased Penetration of Photovoltaic Generation on Smart Grids" Arizona State University, December 2012.

4. S. Eftekharnejad, V. Vittal, G. T. Heydt, B. Keel, and J. Loehr, "Small Signal Stability Assessment of Power Systems With Increased Penetration of Photovoltaic Generation: A Case Study," IEEE Trans. Sustainable Energy., vol. 4, no. 4, pp. 960-967, October 2013

5. P. Kndur, Power System Stability and Control, Tata McGraw- Hill Publishing Company Limited-2001, New Delh.

6. J. H. Wilkinson, The algebraic eigenvalue problem, Clarendon press, Oxford, 1965.

7. Simon P. Teeuwsen, Assessment of the Small Signal Stability of Interconnected Electric Power Systems under Stressed Load Flow Conditions by the Use of Artificial Neural Networks. July 2001.

8. F. Fernandez-Bernal, L. Rouco, P. Centeno, M. Gonzalez, and M. Alonso, "Modeling of photovoltaic plants for power system dynamic studies," IEE Conf. on Power Syst. Management and Control, 17-19 April 2002.

9. H. S. Ko, G. G. Yoon, and W. P. Hong, "Active use of DFIG-based variable-speed windTurbine for voltage regulation at a remote location," IEEE Trans. Power Syst., vol. 22, no. 4, pp. 1916-1925, Nov. 2007.

10. M. A. mehanna, "Optimal location and sizing of photovoltaic generator using simulated annealing algorithm", International Journal of Scientific \& Engineering Research, Volume 7, Issue 5, May-2016. 